\section{Complexité humaine, pathologie et épissage: des signaux pour I'ARN}

Nathalie Matter

\author{
Inserm U.184, Cnrs-LGME, ULP, \\ Institut de génétique et de \\ biologie moléculaire et \\ cellulaire, Parc d'Innovation, \\ 1 , rue Laurent Fries, \\ BP 10142, 67404 IIIkirch Cedex, \\ CU de Strasbourg, France. \\ matter@titus.u-strasbg.fr
}

> Le séquençage du génome humain a montré l'existence de 30000 à 40000 gènes codant pour des protéines $[1,2]$, nombre beaucoup plus faible que ne le laissaient prévoir les esti$(\rightarrow) \mathrm{m} / \mathrm{s} \quad$ mations initiales $(\rightarrow)$. En 2001, $n^{\circ}$ 3, effet, comment expliquer p. 294, la complexité de l'orgap. 309, nisme humain si celui-ci ne dispose que du double de gènes que possède la drosophile ou C. elegans? Cela s'explique principalement par l'existence de mécanismes cellulaires qui permettent la synthèse de plusieurs protéines fonctionnellement différentes à partir d'un même gène, le plus important étant l'épissage alternatif de molécules d'ARN pré-messagers (pré-ARNm). En effet, chez tous les organismes multicellulaires, l'information génétique contenue dans les gènes est fractionnée. Les courtes séquences codantes, nommées exons, sont séparées par de longues séquences non-codantes appelées introns. Après transcription de l'ADN en molécules de pré-ARNm, les introns sont éliminés par un mécanisme d'épissage, rassemblant les exons en une séquence continue assurant la production d'une protéine. Les exons peuvent également être combinés de différentes façons, ce processus est alors appelé épissage alternatif du pré-ARNm. Ainsi, une multitude d'ARNm peuvent être synthétisés à partir d'un pré-ARNm et permettre l'expression de protéines fonctionnellement distinctes à partir d'un gène unique.
Au moins $40 \%$ à $60 \%$ des gènes humains subissent un processus d'épissage alternatif du pré-ARNm [1], mais pour que les variants d'épissage puissent intervenir efficacement dans les processus physiologiques et durant le développement, ils doivent être produits dans la bonne cellule au bon moment. Le processus d'épissage alternatif doit donc être soumis à un contrôle spécifique, dont l'altération est impliquée dans des maladies, comme les maladies neurodégénératives, les myopathies ou certains cancers [3-5]. Pour que l'expression d'isoformes protéiques spécifiques par épissage alternatif soit adaptée aux contraintes spatiales et temporelles, il faut donc postuler un transfert d'information, de l'environnement aux cellules, qui à leur tour transmettront des signaux intracellulaires jusqu'au complexe d'épissage. Cela sous-entend que des facteurs de contrôle de l'épissage alternatif soient induits en réponse à des signaux extracellulaires. C'est effectivement ce que nous avons démontré en identifiant la première protéine régulatrice d'épissage, qui couple une cascade de signalisation cellulaire à l'épissage alternatif du gène CD44 [6].

CD44 comprend dix exons variables, et il existe de multiples formes de la protéine transmembranaire, variants qui jouent un rôle important dans le développement embryonnaire, la réponse immunitaire et le développement tumoral [7-9] $(\rightarrow)$. Des travaux précédents ont permis d'identifier les séquences d'ARN, néces- saires à l'épissage alternatif d'un exon variant (V5) induit par des signaux extra-cellulaires. V5 fait souvent partie intégrante de CD44 dans les cellules cancéreuses durant la progression tumorale et lors de l'activation de lymphocytes T. Dans ces cellules, ainsi que dans celles de lymphome, l'épissage alternatif de V5 est modulé par la voie de signalisation Ras-ERK-MAP (mitogen activated protein)-kinases [10]. Cette cascade enzymatique, impliquant le produit de l'oncogène Ras, est conservée durant l'évolution, et fait le lien entre les récepteurs de la surface cellulaire et leurs cibles régulatrices intracellulaires, c'est en outre une des voies de signalisation majeures au cours du processus oncogénique. Lorsque nous avons cherché les protéines se liant à la séquence ARN de l'exon V5 de CD44, et répondant aux signaux cellulaires, nous avons trouvé une protéine nommée Sam68 (src-associated in mitosis, $68 \mathrm{kDa}$ ). Cette protéine appartient à la famille des protéines STAR (signal transduction and activation of RNA) [11]. En raison de leurs domaines structuraux, il a été suggéré que ces protéines connectent des signaux cellulaires à des processus de maturation de l'ARN. Sam68 joue un rôle dans la progression Gl/S du cycle cellulaire [12]. Sam68 est également un homologue fonctionnel de la protéine Rev du VIH-I qui facilite l'export nucléaire $(\rightarrow) \mathrm{m} / \mathrm{s}$ $1994, n^{\circ} 12$ p. 1282 et 2003, $n^{\circ} 4$, p. 405 de l'ARNm contenant des séquences RRE et permet ainsi l'expression et la replication de virus VIH [13]. 
Des expériences de liaison in vitro et in vivo ont montré que Sam68 liait spécifiquement certains éléments régulateurs de I'ARN de l'exon V5 de CD44. Afin de déterminer si Sam68 peut affecter l'inclusion de l'exon V5 de CD44 dans I'ARNm, en réponse à des signaux cellulaires, un minigène reporteur d'épissage contenant la séquence de l'exon-V5 de CD44 a été cotransfecté avec un vecteur d'expression de Sam68 murin dans des cellules de lymphome $T$. La surexpression de Sam68, seule, n'a pas d'effet significatif sur l'inclusion de l'exon V5 de CD44, mais elle l'augmente significativement en réponse au traitement des cellules avec un ester de phorbol, promoteur de tumeurs, le TPA, qui active la voie de signalisation des Ras-erk-MAP-kinases. Sam68 est phosphorylé lors de l'activation des cellules par le TPA et ce d'une façon Erk-dépendante (Figure 1). De fait, il existe différentes séquences cibles de phosphorylation par la kinase Erk dans la séquence protéique de Sam68, et nous avons montré qu'in vitro, Sam68 est un substrat direct de ErK. La mutation de certains sites cibles de la phosphorylation par Erk, ou l'inhibition de l'expression de Sam68 dans les cellules par des techniques d'anti-sens, inhibent l'activation de l'inclusion de certains exons de CD44.

Nos résultats ont permis d'identifier un premier facteur d'épissage, réglé par la transduction des signaux oncogènes et mitogènes, et phosphorylé directement par Erk [6]. Ainsi, Sam68 peut coupler l'épissage alternatif à une voie de signalisation cellulaire capitale, impliquée dans nombre de processus physiologiques normaux mais aussi pathologiques.

C'est un encouragement à poursuivre l'analyse des mécanismes de régulation de l'épissage alternatif, qui pourrait permettre d'identifier l'altération de protéines responsables du dérèglement de l'épissage dans de nombreuses maladies humaines [14]. $\diamond$

Control of RNA splicing

by extracellular signals

\section{RÉFÉRENCES}

1. International human genome sequencing consortium Initial sequencing and analysis of the human genome. Nature 2001; 409: 860-921.

2. Venter JC, Adams MD, Myers $\varepsilon W$, et al. The sequence of the human genome. Science 2001 ; 291 : 1304-51.

3. Cooper TA, Mattox W. The regulation of splice-site selection, and its role in human disease. Am J Hum Genet 1997; 61: 259-66.

4. Dredge BK, Polydorides AD, Darnell RB. The splice of life: alternative splicing and neurological disease. Nat Rev Neurosci 2001 ; 2:43-50.

5. Charlet BN, Savkurs RS, Singh G, Philips AV, Grice $\varepsilon A$, Cooper TA. Loss of the muscle-specific chloride channel in type 1 myotonic dyxtrophy due to misregulated alternative splicing. Mol Cell 2002; $10: 45-53$.
6. Matter N, Herrlich P, König H. Signal-dependent regulation of splicing via phosphorylation of Sam68. Nature 2002; 420: 691-5.

7. Arch R, Wirth K, Hofmann $M$, et al. Participation in normal immune responses splice variant of CD44. Science 1992; 257 : 682-5.

8. Cooper DL, Dougherty GJ. To metastasize or not? sites. Nat Med 1995; 1 : 635-7.

9. Sherman L, Wainright $D$, Ponta H, Herrlich P. A splice variant of CD44 expressed in the apical ectodermal ridge presents fibroblast growth factors to limb mesenchyme and is required for limb outremonts. Genes Dev 1998; 12: 1058-71.

10. Weg-Remers S, Ponta H, Herrlich P, König $H$. Regulation of alternative pre-mRNA splicing by the ERK-MAP-kinase pathway. EMBO J 2001; 20 : 4194-203.

11. Vernet C, Artzt K. STAR, a of a metastasis-inducing Selection of CD44 splice gene family involved in signal transduction and activation of RNA. Trends Genet 1997; 13 : 470-84.

12. Barlat I, Maurier F, Duschene M, Guitard $\varepsilon$, Tocque B, Schweighoffer $F$. A role for Sam68 in cell cycle progression antagonized by a spliced variant within the $\mathrm{KH}$ 272: 3129-32.

13. Reddy TR, XU W, Mau JK, et al. Inhibition of HIV replication by dominant negative mutants of Sam68, a functional homolog of HIV-1. Rev Nat Med 1999; 5: 635-42.

14. Nissim-Rafinia M, Kerem B. Splicing regulation as a potential genetic modifier. Trends Genet 2002; 18 : 123-7. domain. J Biol Chem 1996;

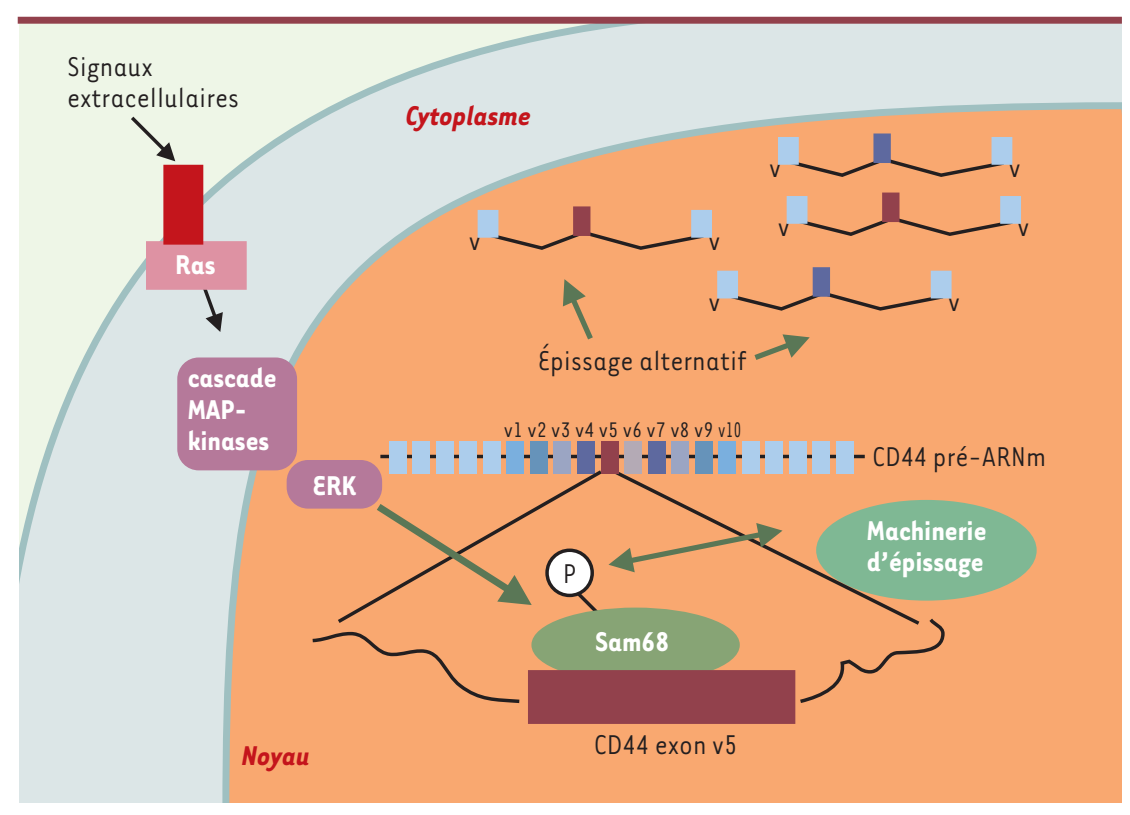

Figure 1. Transduction de signaux depuis la surface cellulaire à la machinerie d'épissage. Les signaux induits à la surface cellulaire après la fixation d'un ligand extracellulaire à son récepteur (rouge) sont véhiculés par la cascade des Ras-ErK-MAP-kinases jusqu'au noyau cellulaire où Sam68 est phosphorylé par la kinase $\varepsilon r k$. Ainsi activé, Sam68 augmente l'inclusion de l'exon V5 dans les transcrits de CD44 durant l'épissage alternatif. 